\title{
28. ABUNDANCE PATTERNS OF TUNICATE SPICULES AT THE GREAT BARRIER REEF- QUEENSLAND PLATEAU TRANSECT SITES: IMPLICATIONS FOR DOWNSLOPE TRANSPORT AND EARLY PLEISTOCENE INITIATION OF THE CENTRAL GREAT BARRIER REEF ${ }^{1}$
}

\author{
Wuchang Wei ${ }^{2}$
}

\begin{abstract}
The abundance patterns of tunicate spicules are documented for the Pliocene-Pleistocene sediments at seven sites along the Great Barrier Reef-Queensland Plateau transect. The spatial distribution pattern indicates that tunicate spicules were limited to waters shallower than $900 \mathrm{~m}$. The occurrences of tunicate spicules at Sites 822 and 823 that are deeper than $900 \mathrm{~m}$ are ascribed to downslope transport, and their distribution patterns can be used to monitor downslope transport processes. The first common occurrence of tunicate spicules at Sites 822 and 823 around 1.6 Ma may suggest the initiation of the central Great Barrier Reef at this time. The morphology of tunicate spicules varies greatly and appears to be gradational among different forms. Older tunicate assemblages are less diverse than those in younger sediments, presumably because of diagenesis. Tunicate spicules do not appear to be a promising biostratigraphic tool for the Pliocene-Pleistocene.
\end{abstract}

\section{INTRODUCTION}

Tunicates (Phylum Chordata, Class Assidacea, Genus Didemnum) are benthic organisms occurring in solitary and colonial forms and are common throughout the world's oceans. Tunicates containing large amounts of calcium carbonate spicules are found mainly in the Didemnidae and Pyuridae families. Tunicate spicules are aragonitic and are limited to waters shallower than 200 to $500 \mathrm{~m}$ (Heckel, 1973; Milliman, 1974). They usually prefer a hard substratum on which they build up colonies. Abundant tunicate spicules (a few percent of the sediments) have been reported only in tropical waters, such as the Bahamas (Newell et al., 1959; Purdy, 1963) and the Great Barrier Reef area (Heckel, 1973). The latter author provided information about the distribution of tunicate spicules on the seafloor and in Miocene-Pliocene sediments recovered from two industrial wells in the Great Barrier Reef region.

During Leg 133, abundant tunicate spicules were recovered at Sites 819 through 823 on the Great Barrier Reef-Queensland Plateau transect (Fig. 1). The objectives of this study are (1) to provide a detailed documentation of the distribution of tunicate spicules through time and space (along the transect); (2) to infer downslope transport of sediments and initiation of reef growth in the central Great Barrier Reef; and (3) to report observations about the taxonomy of tunicate spicules and to investigate their possible biostratigraphic utility. The second objective is based on the assumption that tunicate spicules found in waters much deeper than $500 \mathrm{~m}$ were derived from downslope transport and that abundant tunicate spicules are derived from the reef areas.

\section{MATERIAL AND METHODS}

Generally, two samples per core were analyzed for this study. Smear slides were made directly from unprocessed samples and were examined with a light microscope. Specimens of tunicate spicules were counted at a magnification of $190 \times$ in 10 fields of view along random traverses of a slide. Most slides are fairly uniform in thickness, because they were prepared by the same person. For some slides that are obviously thicker or thinner than the average, the total number of specimens counted has been corrected for the average. One hun-

\footnotetext{
'McKenzie, J.A., Davies, P.J., Palmer-Julson, A., etal., 1993. Proc. ODP. Sci. Results, 133: College Station, TX (Ocean Drilling Program).

${ }^{2}$ Scripps Institution of Oceanography, University of California, San Diego, La Jolla, CA 92093-0215, U.S.A.
}

dred specimens in 10 fields of view were estimated as equivalent to $4 \%$ of the sediment. The abundance data of tunicate spicules from ODP Sites 820 through 823 are provided in Table 1. Data from ODP Sites 811 and 824 and DSDP Site 209 are not listed in Table 1, because these sites do not yield tunicate spicules in the Pleistocene.

Tunicate spicules were photographed in both the light microscope and the scanning electron microscope (SEM). Preparation of samples for SEM microscopy followed standard procedures. Photomicrographs are shown in Plates 1 and 2. Age information was taken from Wei and Gartner (this volume). Detailed site descriptions can be found in Davies, McKenzie, Palmer-Julson, et al. (1991).

\section{RESULTS}

The distribution of tunicate spicules along the Great Barrier ReefQueensland Plateau transect is shown in Figure 2. Site 821, which is shallower and closer to the Great Barrier Reef than other sites, contained the most abundant tunicate spicules (up to $8 \%$ of the sediment). Sites 820,822 , and 823 yielded similar abundances; Sites 209, 811, and 824 on the Queensland Plateau contained no tunicate spicules. The abundance patterns at Sites 820 through 823 have some similarities and show a general decrease in abundance downhole. The fluctuations in abundance at these sites are also somewhat similar, although the details cannot be matched from site to site. Figure 2 also indicates that tunicate spicules became common only after about $1.6 \mathrm{Ma}$; before this time, tunicate spicules were virtually absent (see Sites 822 and 823).

\section{DISCUSSION}

As stated above, the Queensland Plateau sites, which are located in water depths greater than $900 \mathrm{~m}$, did not yield tunicate spicules. This result supports the notion that tunicate spicules are limited to waters shallower than 200 to $500 \mathrm{~m}$ (Heckel, 1973; Milliman, 1974). Interestingly, Sites 822 (water depth $=955 \mathrm{~m}$ ) and 823 (water depth $=1649$ $\mathrm{m})$ yielded abundant tunicate spicules. These tunicate spicules are ascribed to downslope transport because they could not live at these water depths. The abundance patterns of tunicate spicules at Sites 822 and 823 , therefore, offer a useful means to monitor the process of downslope sediment transport. Figure 2 suggests that there has been continuous downslope transport for at least the last $1.6 \mathrm{~m} . \mathrm{y}$. This is also suggested by benthic foraminiferal data and sedimentological data, particularly at Site 823 , where virtually the entire sediment sequence is ridden with debris flows (Davies, McKenzie, Palmer-Julson, et al., 


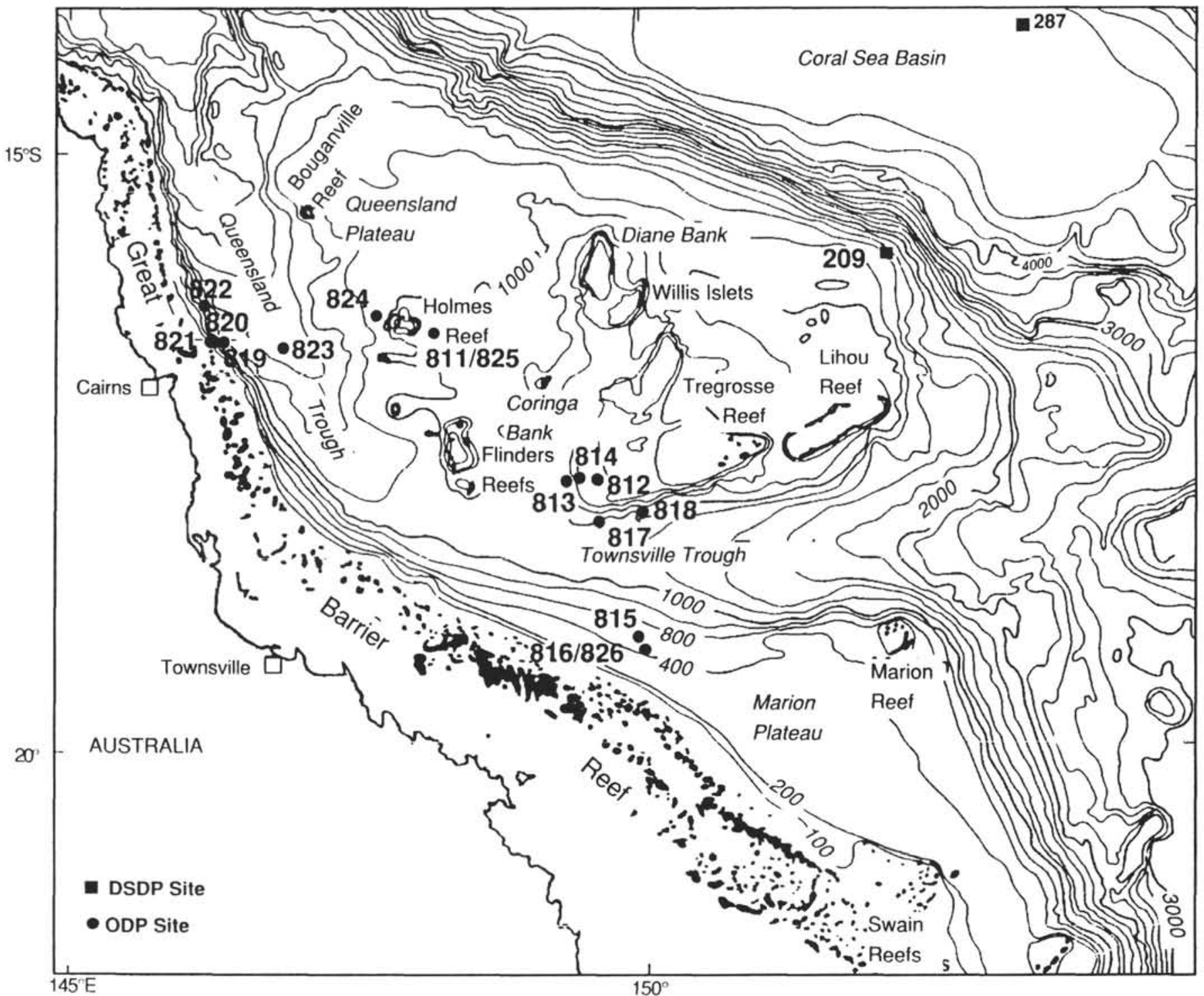

Figure 1. Map of northeastern Australian margin showing Leg 133 drill sites. Bathymetry in meters.

1991). On the other hand, calcareous nannofossil assemblages in these sediments do not show a mixture of species of different ages (Wei and Gartner, this volume). This means that downslope transport has virtually been a penecontemporaneous redeposition process, which did not involve sediments of appreciably older ages.

Downslope transport apparently was also the cause for the abundant tunicate spicules found in the Miocene-Pliocene turbidites at Site 210 (Burns, Andrew, et al., 1973). This site lies in the Coral Sea Basin at a water depth of $4643 \mathrm{~m}$ and shows a similar assemblage of tunicate spicules (Edwards, 1973) as found in this study, whereas Site 209, at a water depth of $1428 \mathrm{~m}$ on the north margin of the Queensland Plateau, yielded no tunicate spicules (Fig. 2). This suggests that the shelf north of the Coral Sea supplied the tunicate spicules found in the turbidites at Site 210 and that the Queensland Plateau did not supply appreciable amounts of tunicate spicules for downslope transport.

Dissolution of tunicate spicules can affect distribution patterns and may be partially responsible for a general decrease in abundance downhole. However, the first common occurrence of tunicate spicules at about $1.6 \mathrm{Ma}$ at Sites 822 and 823 (Fig. 2) cannot be ascribed to diagenesis because it is virtually synchronous, whereas the depths of common occurrence are different $(\sim 320$ mbsf at Site 822 vs. $\sim 160$ $\mathrm{mbsf}$ at Site 823 ). Data in Figure 2 suggest that by about $1.6 \mathrm{Ma}$, large quantities of tunicates had been produced in the shelf areas, presumably as a result of the initiation of the central Great Barrier Reef, which provided large areas of hard substrate favoring the growth of tunicates. More abundant tunicate spicules at Site 821, which is closer to the Great Barrier Reef than other sites, also suggests that tunicate spicules came mostly from the direction of the Great Barrier Reef. The inferred age for the initiation of the central Great Barrier Reef is consistent with the data of Montaggioni and Venec-Peyre (this volume), who found tropical, reef-derived foraminifers throughout the sequence at Site 821 . The maximum age for the initiation of reef growth, however, cannot be determined at Site 821, which reached sediment younger than $1.48 \mathrm{Ma}$.

The tunicate spicules observed in this study are generally 40 to 100 $\mu \mathrm{m}$ in size. The number of rays in each specimen varies greatly, ranging from 3 to more than 50 (Pls. 1 and 2). These tunicate spicules also appear to grade from one form to another and are difficult to separate into different species. Tunicate spicule assemblages are diverse in the youngest sediment, as can be seen in Plates 1 and 2 . Diversity decreases downhole. This is ascribed to diagenesis. No noticeably different tunicate spicules were observed deeper in the hole than those observed in the younger sediments. This indicates that tunicate spicules generally are not useful for biostratigraphy for the Pliocene-Pleistocene. 
Table 1. Abundance of tunicate spicules along the Great Barrier Reef-Queensland Plateau transect.

\begin{tabular}{|c|c|c|c|c|c|c|c|c|c|c|c|}
\hline $\begin{array}{l}\text { Hole } \\
821 \mathrm{~A}\end{array}$ & $\begin{array}{l}\text { Depth } \\
\text { (mbsf) }\end{array}$ & Num. & $\begin{array}{l}\text { Hole } \\
820 \mathrm{~B}\end{array}$ & $\begin{array}{l}\text { Depth } \\
\text { (mbsf) }\end{array}$ & Num. & $\begin{array}{l}\text { Hole } \\
822 \mathrm{~A}\end{array}$ & $\begin{array}{l}\text { Depth } \\
\text { (mbsf) }\end{array}$ & Num. & $\begin{array}{l}\text { Hole } \\
823 \mathrm{~A}\end{array}$ & $\begin{array}{l}\text { Depth } \\
\text { (mbsf) }\end{array}$ & Num. \\
\hline $1 \mathrm{H}-1,111$ & 1.12 & 105 & $1 \mathrm{H}-1,112$ & 1.12 & 56 & IH-CC & 0.90 & 112 & $1 \mathrm{H}-1,115$ & 1.15 & 61 \\
\hline $1 \mathrm{H}-3,112$ & 4.12 & 141 & $1 \mathrm{H}-3,112$ & 4.12 & 71 & $2 \mathrm{H}-2,112$ & 3.52 & 47 & $1 \mathrm{H}-4,115$ & 5.65 & 26 \\
\hline $2 \mathrm{H}-1,111$ & 5.51 & 164 & $2 \mathrm{H}-1,112$ & 8.32 & 63 & $2 \mathrm{H}-5,112$ & 8.02 & 105 & $2 \mathrm{H}-2,115$ & 8.45 & 12 \\
\hline $2 \mathrm{H}-3,111$ & 8.51 & 102 & $2 \mathrm{H}-4,112$ & 12.82 & 41 & $3 \mathrm{H}-1,112$ & 11.52 & 60 & $2 \mathrm{H}-6,115$ & 14.45 & 14 \\
\hline $3 \mathrm{H}-1,111$ & 15.01 & 77 & $3 \mathrm{H}-1,112$ & 17.82 & 48 & $3 \mathrm{H}-4,112$ & 16.02 & 63 & $3 \mathrm{H}-4,115$ & 20.95 & 20 \\
\hline $3 \mathrm{H}-4,111$ & 19.51 & 97 & $3 \mathrm{H}-4,112$ & 22.32 & 44 & $4 \mathrm{H}-1,113$ & 21.03 & 32 & $4 \mathrm{H}-1,115$ & 25.95 & 13 \\
\hline $4 \mathrm{H}-1,111$ & 24.51 & 140 & $4 \mathrm{H}-1,112$ & 27.32 & 76 & $4 \mathrm{H}-4,113$ & 22.53 & 51 & $4 \mathrm{H}-4,115$ & 30.45 & 31 \\
\hline $4 \mathrm{H}-4,111$ & 29.01 & 205 & $4 \mathrm{H}-4,112$ & 31.82 & 66 & $5 \mathrm{H}-1,112$ & 30.52 & 56 & $5 \mathrm{H}-1,115$ & 35.45 & 0 \\
\hline $5 \mathrm{H}-1,111$ & 34.01 & 89 & $5 \mathrm{H}-1,112$ & 36.82 & 58 & $5 \mathrm{H}-4,112$ & 35.02 & 61 & $5 \mathrm{H}-5,115$ & 41.45 & 1 \\
\hline $5 \mathrm{H}-4,111$ & 38.51 & 60 & $5 \mathrm{H}-4,112$ & 41.32 & 69 & $6 \mathrm{H}-1,113$ & 40.03 & 43 & $6 \mathrm{H}-1,115$ & 44.95 & 13 \\
\hline $6 \mathrm{H}-1,111$ & 43.51 & 105 & $6 \mathrm{H}-1,112$ & 46.32 & 61 & $6 \mathrm{H}-4,113$ & 44.53 & 55 & $6 \mathrm{H}-4,115$ & 49.45 & 17 \\
\hline $6 \mathrm{H}-4,111$ & 48.01 & 76 & $6 \mathrm{H}-4,112$ & 50,82 & 90 & $7 \mathrm{H}-\mathrm{I}, 112$ & 49.52 & 70 & $7 \mathrm{H}-1,115$ & 54.45 & 21 \\
\hline $7 \mathrm{H}-5,111$ & 59.01 & 95 & $7 \mathrm{H}-1,110$ & 55.80 & 38 & $7 \mathrm{H}-4,112$ & 54.02 & 31 & $7 \mathrm{H}-4,115$ & 58.95 & 22 \\
\hline $8 \mathrm{H}-1,112$ & 62.52 & 135 & $7 \mathrm{H}-4,110$ & 60.30 & 38 & $8 \mathrm{H}-1,111$ & 59.01 & 48 & $8 \mathrm{H}-1,115$ & 63.95 & 13 \\
\hline $8 \mathrm{H}-4,112$ & 67.02 & 150 & $8 \mathrm{H}-1,112$ & 65.32 & 19 & $8 \mathrm{H}-4,111$ & 63.51 & 41 & $8 \mathrm{H}-4,115$ & 68.45 & 43 \\
\hline $9 \mathrm{H}-1,112$ & 72.02 & 129 & $8 \mathrm{H}-4,112$ & 69.82 & 25 & $9 \mathrm{H}-2,113$ & 69.12 & 28 & $9 \mathrm{H}-1,115$ & 73.45 & 24 \\
\hline $9 \mathrm{H}-4,112$ & 76.52 & 127 & $9 \mathrm{H}-1,112$ & 74.82 & 49 & $9 \mathrm{H}-5,113$ & 73.62 & 41 & $9 \mathrm{H}-4,115$ & 77.95 & 27 \\
\hline $10 \mathrm{H}-1,112$ & 81.51 & 110 & $9 \mathrm{H}-4,112$ & 79.32 & 55 & $10 \mathrm{H}-2,114$ & 78.74 & 22 & $10 \mathrm{H}-2,115$ & 84.45 & 25 \\
\hline $11 \mathrm{H}-1,112$ & 91.02 & 78 & $10 \mathrm{H}-1,112$ & 84.32 & 37 & $10 \mathrm{H}-4,124$ & 81.74 & 51 & $10 \mathrm{H}-5,115$ & 88.95 & 5 \\
\hline $11 \mathrm{H}-4,112$ & 95.52 & 50 & $10 \mathrm{H}-4,112$ & 88.82 & 45 & $11 \mathrm{H}-1,111$ & 87.51 & 60 & $11 \mathrm{H}-1,116$ & 92.46 & 4 \\
\hline $12 \mathrm{H}-1,112$ & 100.52 & 38 & $11 \mathrm{H}-1,111$ & 93.81 & 45 & $11 \mathrm{H}-4,111$ & 92.01 & 13 & $11 \mathrm{H}-4,116$ & 96.96 & 5 \\
\hline $12 \mathrm{H}-4,112$ & 105.02 & 60 & $11 \mathrm{H}-4,111$ & 98.31 & 37 & $12 X-1,111$ & 97.01 & 31 & $12 \mathrm{H}-1,116$ & 101.96 & 20 \\
\hline $13 \mathrm{H}-1,112$ & 111.52 & 49 & $12 \mathrm{H}-1,110$ & 103.30 & 29 & $12 X-4,111$ & 101.51 & 41 & $12 \mathrm{H}-4,116$ & 106.46 & 6 \\
\hline $13 \mathrm{H}-4,112$ & 114.52 & 46 & $12 \mathrm{H}-4,110$ & 107.80 & 36 & $13 \mathrm{X}-1,111$ & 106.81 & 89 & $13 \mathrm{H}-1,113$ & 111,46 & 21 \\
\hline $14 \mathrm{H}-1,112$ & 119.52 & 81 & $13 \mathrm{H}-1,110$ & 112.80 & 22 & $13 X-4,106$ & 111.26 & 43 & $14 \mathrm{H}-\mathrm{CC} 13$ & 2.10 & 11 \\
\hline $14 \mathrm{H}-4,112$ & 124.02 & 41 & $13 \mathrm{H}-4,110$ & 117.30 & 67 & $14 \mathrm{X}-1,105$ & 116.45 & 47 & $15 \mathrm{H}-\mathrm{CCl} 4$ & 1.70 & 23 \\
\hline $15 \mathrm{H}-1,112$ & 129.02 & 29 & $14 \mathrm{H}-1,110$ & 122.30 & 58 & $14 X-4,105$ & 120.95 & 44 & $16 \mathrm{H}-2,115$ & 144.36 & 24 \\
\hline $15 \mathrm{H}-4,112$ & 133.52 & 70 & $1 \mathrm{H}-1,110$ & 131.80 & 42 & $15 X-1,112$ & 126.12 & 25 & $17 \mathrm{X}-\mathrm{CC}$ & 161.10 & 0 \\
\hline $16 \mathrm{H}-1,112$ & 138.52 & 38 & $15 \mathrm{H}-4,110$ & 136.30 & 39 & $15 X-4,112$ & 130.62 & 40 & $18 X-2,115$ & 163.75 & 0 \\
\hline $16 \mathrm{H}-4,112$ & 143.02 & 77 & $16 \mathrm{~V}-1,110$ & 141.30 & 30 & $16 \mathrm{X}-1,119$ & 135.79 & 41 & $18 X-5,115$ & 168.25 & 0 \\
\hline $17 X-1,112$ & 147.02 & 104 & $17 \mathrm{~V}-1,112$ & 151.82 & 14 & $17 X-1,112$ & 145.42 & 42 & $19 \mathrm{X}-\mathrm{CC} 18$ & 0.50 & 1 \\
\hline $17 X-4,112$ & 151.52 & 67 & $17 \mathrm{~V}-4,112$ & 156.32 & 24 & $18 X-2,112$ & 156.62 & 21 & $20 \mathrm{X}-\mathrm{CC} 19$ & 0.20 & 0 \\
\hline $18 X-1,112$ & 156.72 & 47 & $18 \mathrm{X}-1,112$ & 161.32 & 22 & $19 X-1,112$ & 164.82 & 6 & $21 \mathrm{X}-\mathrm{CC} 19$ & 9.80 & 1 \\
\hline $18 X-5,112$ & 162.72 & 21 & $18 X-4,112$ & 165.82 & 28 & $20 \mathrm{X}-1,112$ & 174.42 & 13 & $22 \mathrm{X}-\mathrm{CC} 20$ & 9.10 & 0 \\
\hline $19 X-1,112$ & 166.32 & 12 & $19 X-1,112$ & 166.32 & 14 & $21 X-1,112$ & 183.72 & 8 & $23 \mathrm{X}-\mathrm{CC} 21$ & 8.70 & 0 \\
\hline $19 \mathrm{X}-4,112$ & 170.82 & 20 & $19 X-4,112$ & 170.82 & 12 & $22 \mathrm{X}-1,112$ & 193.92 & 33 & $24 X-3,137$ & 223.07 & 1 \\
\hline $20 \mathrm{X}-2,112$ & 177.52 & 32 & $20 \mathrm{X}-1,112$ & 175.92 & 64 & $23 \mathrm{X}-1,112$ & 202.63 & 20 & $24 X-5,137$ & 225.89 & 0 \\
\hline $20 X-5,112$ & 182.02 & 28 & $20 X-4,112$ & 180.42 & 50 & $24 X-1,37$ & 212.32 & 39 & $25 \mathrm{X}-1,137$ & 229.37 & 0 \\
\hline $21 X-2,104$ & 187.14 & 27 & $21 X-1,112$ & 185.22 & 50 & $25 X-1,37$ & 221.27 & 8 & $25 X-4,140$ & 233.90 & 1 \\
\hline $21 X-5,105$ & 191.65 & 1 & $21 X-4,112$ & 189.72 & 42 & $26 \mathrm{X}-1,112$ & 231.62 & 25 & $26 \mathrm{X}-1,136$ & 239.06 & 1 \\
\hline $22 X-1,110$ & 195.00 & 14 & $22 \mathrm{X}-1,112$ & 194.82 & 46 & $27 X-2,112$ & 242.36 & 10 & $26 X-4,136$ & 243.56 & 0 \\
\hline $22 X-5,110$ & 201.00 & 12 & $22 X-4,112$ & 199.32 & 20 & $28 \mathrm{X}-1,112$ & 250.92 & 3 & $27 \mathrm{X}-\mathrm{CC} 25$ & 7.00 & 1 \\
\hline $23 \mathrm{X}-1,111$ & 204.61 & 28 & $23 \mathrm{X}-1,111$ & 204.51 & 40 & $29 X-2,112$ & 260.96 & 3 & $28 \mathrm{X}-\mathrm{CC} 26$ & 6.70 & 0 \\
\hline $23 X-5,111$ & 210.61 & 27 & $23 X-4,114$ & 209.04 & 34 & $30 X-1,112$ & 270.22 & 25 & $29 \mathrm{X}-\mathrm{CC} 27$ & 6.30 & 0 \\
\hline $24 X-1,111$ & 213.91 & 29 & $24 \mathrm{X}-1,112$ & 213.92 & 37 & $31 X-2,112$ & 280.48 & 12 & $30 \mathrm{X}-\mathrm{CC} 28$ & 6.00 & 2 \\
\hline $24 X-5,111$ & 219.91 & 32 & $24 X-4,112$ & 218.42 & 24 & $32 X-2,112$ & 291.02 & 10 & $31 \mathrm{X}-\mathrm{CC} 29$ & 5.60 & 0 \\
\hline $25 \mathrm{X}-1,111$ & 223.61 & 29 & $25 \mathrm{X}-1,121$ & 223.71 & 46 & $33 X-1,112$ & 299.22 & 41 & $32 \mathrm{X}-\mathrm{CC} 30$ & 5.30 & 3 \\
\hline $25 X-5,111$ & 229.61 & 31 & $25 \mathrm{X}-3,121$ & 226.71 & 3 & $34 \mathrm{X}-1,112$ & 308.92 & 34 & $33 \mathrm{X}-\mathrm{CC} 31$ & 4.90 & 1 \\
\hline $26 \mathrm{X}-1,111$ & 233.31 & 23 & $27 \mathrm{X}-1,112$ & 242.92 & 4 & $35 \mathrm{X}-1,112$ & 318.62 & 2 & $34 \mathrm{X}-\mathrm{CC} 32$ & 4.60 & 0 \\
\hline $26 \mathrm{X}-4,111$ & 237.81 & 12 & $27 X-4,112$ & 247.42 & 6 & $36 \mathrm{X}-1,112$ & 328.12 & 1 & & & \\
\hline $27 \mathrm{X}-1,122$ & 243.02 & 29 & $28 \mathrm{X}-1,112$ & 252.22 & 3 & $37 X-2,112$ & 338.58 & 3 & & & \\
\hline $27 X-4,116$ & 247.46 & 16 & $28 X-4,112$ & 256.72 & 16 & $38 \mathrm{X}-1,111$ & 347.41 & 1 & & & \\
\hline $28 \mathrm{X}-1,112$ & 252.52 & 22 & $29 \mathrm{X}-1,115$ & 261.95 & 2 & $39 \mathrm{X}-\mathrm{CC}$ & 365.50 & 0 & & & \\
\hline $28 \mathrm{X}-4,112$ & 257.02 & 8 & $29 X-4,112$ & 266.42 & 2 & $40 X-2,112$ & 368.12 & 0 & & & \\
\hline $29 X-2,116$ & 263.76 & 3 & $30 X-1,113$ & 271.53 & 3 & $41 X-2,112$ & 377.04 & 0 & & & \\
\hline $30 X-2,112$ & 273.42 & 9 & $32 X-1,112$ & 290.82 & 0 & $42 X-2,112$ & 387.42 & 1 & & & \\
\hline $31 X-2,112$ & 283.02 & 0 & $33 \mathrm{X}-1,112$ & 300.52 & 1 & $43 \mathrm{X}-1,112$ & 395.62 & 3 & & & \\
\hline $31 X-5,112$ & 287.52 & 0 & $33 \mathrm{X}-4,112$ & 305.02 & 20 & $44 X-1,112$ & 401.12 & 11 & & & \\
\hline $33 \mathrm{X}-1,112$ & 300.82 & 3 & $35 \mathrm{X}-1,112$ & 319.92 & 7 & $45 X-1,112$ & 406.02 & 2 & & & \\
\hline $34 \mathrm{X}-1,112$ & 310.52 & 2 & $36 \mathrm{X}-1,112$ & 329.62 & 14 & $46 \mathrm{X}-1,112$ & 415.71 & 0 & & & \\
\hline $34 X-4,112$ & 315.02 & 7 & $36 X-4,112$ & 334.12 & 41 & $47 X-1,112$ & 425.41 & 0 & & & \\
\hline $35 X-1,116$ & 320.16 & 0 & $37 X-2,112$ & 340.82 & 27 & $47 X-4,111$ & 429.91 & 1 & & & \\
\hline $35 X-4,116$ & 324.66 & 1 & $37 X-4,112$ & 343.82 & 14 & 47X-CC & 433.90 & 3 & & & \\
\hline $36 X-2,112$ & 330.38 & 2 & $38 \mathrm{X}-1,112$ & 349.02 & 41 & & & & & & \\
\hline $36 X-5,112$ & 334.88 & 5 & $38 X-3,112$ & 352.02 & 0 & & & & & & \\
\hline $37 X-2,112$ & 340.92 & 6 & $39 X-1,112$ & 358.62 & 27 & & & & & & \\
\hline $37 X-5,112$ & 345.42 & 3 & $40 X-1,112$ & 363.82 & 9 & & & & & & \\
\hline $38 \mathrm{X}-2,112$ & 350.52 & 1 & $41 X-1,112$ & 368.22 & 1 & & & & & & \\
\hline $38 X-5,112$ & 355.02 & 2 & $41 X-3,112$ & 371.22 & 0 & & & & & & \\
\hline $39 \mathrm{X}-2,112$ & 360.12 & 3 & $42 X-1,112$ & 377.82 & 0 & & & & & & \\
\hline $39 X-5,112$ & 364.62 & 5 & $42 X-4,112$ & 382.32 & 1 & & & & & & \\
\hline $40 X-2,112$ & 369.82 & 4 & $44 X-1,112$ & 397.12 & $i$ & & & & & & \\
\hline $40 X-5,112$ & 374.32 & 0 & & & & & & & & & \\
\hline $41 X-2,112$ & 379.42 & 6 & & & & & & & & & \\
\hline $41 X-5,112$ & 383.92 & 7 & & & & & & & & & \\
\hline $42 X-2,112$ & 389.03 & 5 & & & & & & & & & \\
\hline $42 X-5,112$ & 393.53 & 3 & & & & & & & & & \\
\hline $43 X-2,112$ & 398.72 & 3 & & & & & & & & & \\
\hline
\end{tabular}



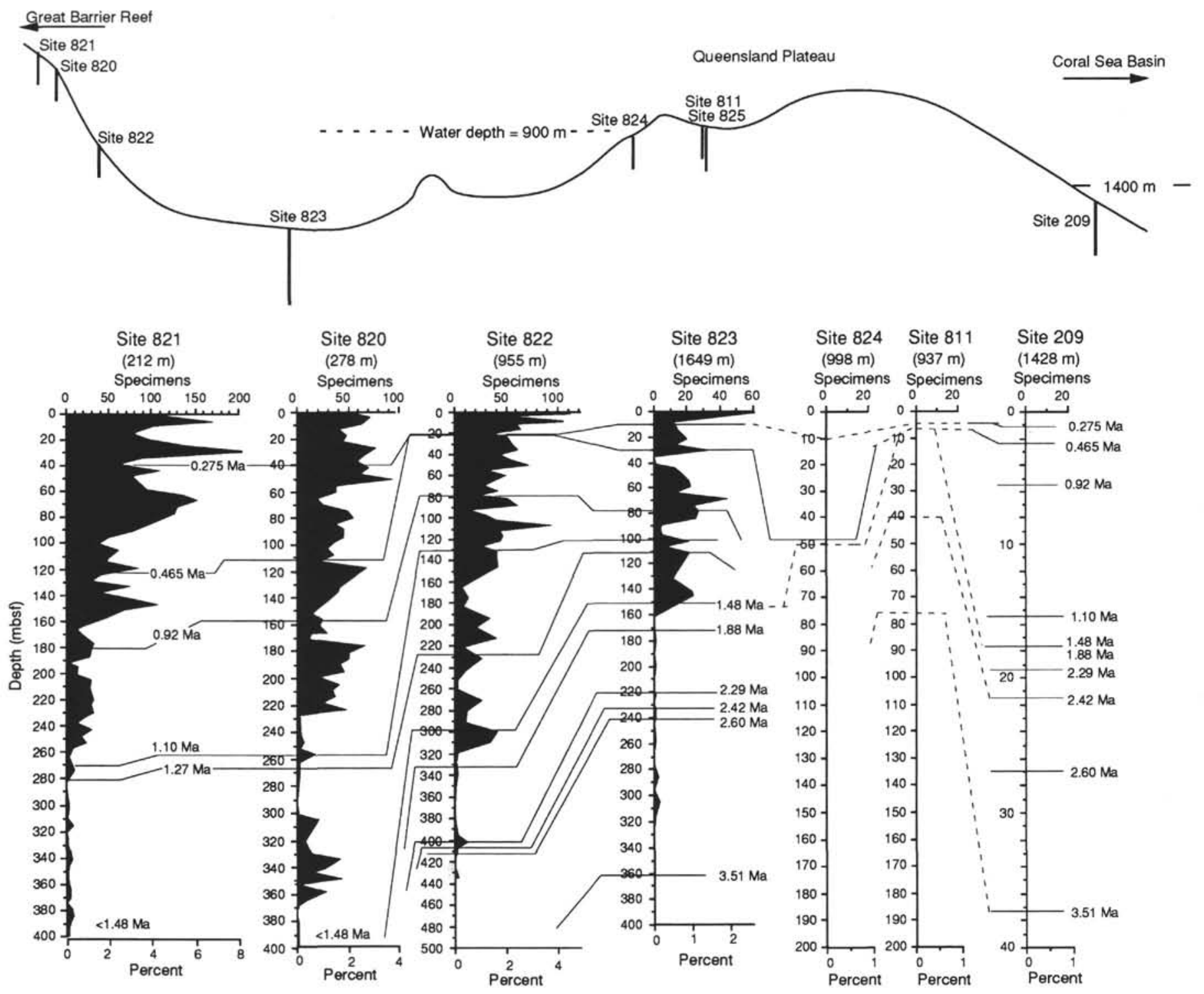

Figure 2. Schematic cross section showing sites investigated for abundance patterns of tunicate spicules (upper panel); abundances of tunicate spicules for Sites 821 through 824 and 209 are shown in the lower panel.

\section{CONCLUSIONS}

1. The distribution of tunicate spicules along the Great Barrier Reef-Queensland Plateau transect indicates that these organisms lived in waters shallower than $900 \mathrm{~m}$. This agrees with previous reports that tunicate spicules are generally limited to waters shallower than 200 to $500 \mathrm{~m}$.

2. The occurrences of tunicate spicules at Sites 822 and 823 , which are deeper than $900 \mathrm{~m}$, are ascribed to downslope transport from the shelf of the Great Barrier Reef. Their distribution patterns thus offer a useful means of monitoring downslope transport processes.

3. The first common occurrence of tunicate spicules at Sites 822 and 823 around 1.6 Ma may suggest the initiation of the central Great Barrier Reef at this time.

4. The morphology of tunicate spicules varies greatly and appears to be gradational among different forms. Tunicate assemblages are more diverse in younger sediments than in older sediments, presumably because of diagenesis. Tunicate spicules do not appear to be a promising biostratigraphic tool for the Pliocene-Pleistocene.

\section{ACKNOWLEDGMENTS}

I thank the crew of the JOIDES Resolution, the ODP technical staff, and the Leg 133 Scientific Party, particularly P. Davies, J. McKenzie (Co-Chief Scientists), and A. Palmer-Julson (Staff Scientist) for an enjoyable and very successful cruise. I also thank K. von Salis Perch-Nielsen and S.W. Wise for critical reviews and helpful suggestions. Financial support was provided by NSF Grant DPP9118480 (to S.W. Wise), USSAC grants, and NSF Grant OCE91-15786 (to W. Wei).

\section{REFERENCES}

Burns, R.E., Andrew, J.E., et al., 1973. Init. Repts. DSDP, 21: Washington(U.S. Govt. Printing Office).

\footnotetext{
- Abbreviations for names of organizations and publications in ODP reference lists follow the style given in Chemical Abstracts Service Source Index (published by American Chemical Society).
} 
Davies, P.J., McKenzie, J.A., Palmer-Julson, A., et al., 1991. Proc. ODP, Init. Repts., 133: College Station, TX (Ocean Drilling Program).

Edwards, A., 1973. Calcareous nannofossils. In Burns, R.E., Andrew, J.E., et al., Init. Repts. DSDP, 21: Washington (U.S. Govt. Printing Office), 641-691.

Heckel, H., 1973. Late Oligocene to Recent nannoplankton from the Capricorn Basin (Great Barrier Reef area). Geol. Surv. Queensland, Palaeontol. Pap., 33:1-24.

Milliam, J.D., 1974. Recent Sedimentary Carbonates. I. Marine Carbonates: New York (Springer Verlag).
Newell, N.D., Imbrie, J., Purdy, E.G., and Thurber, D.L., 1959. Organism communities and bottom facies, Great Bahama Bank. Bull. Am. Mus. Nat. Hist., 117:177-228.

Purdy, E.G., 1963. Recent calcium carbonate facies of the Great Bahama Bank. J. Geol., 71:334-355.

Date of initial receipt: 2 March 1992

Date of acceptance: 10 December 1992

Ms 133SR-278 

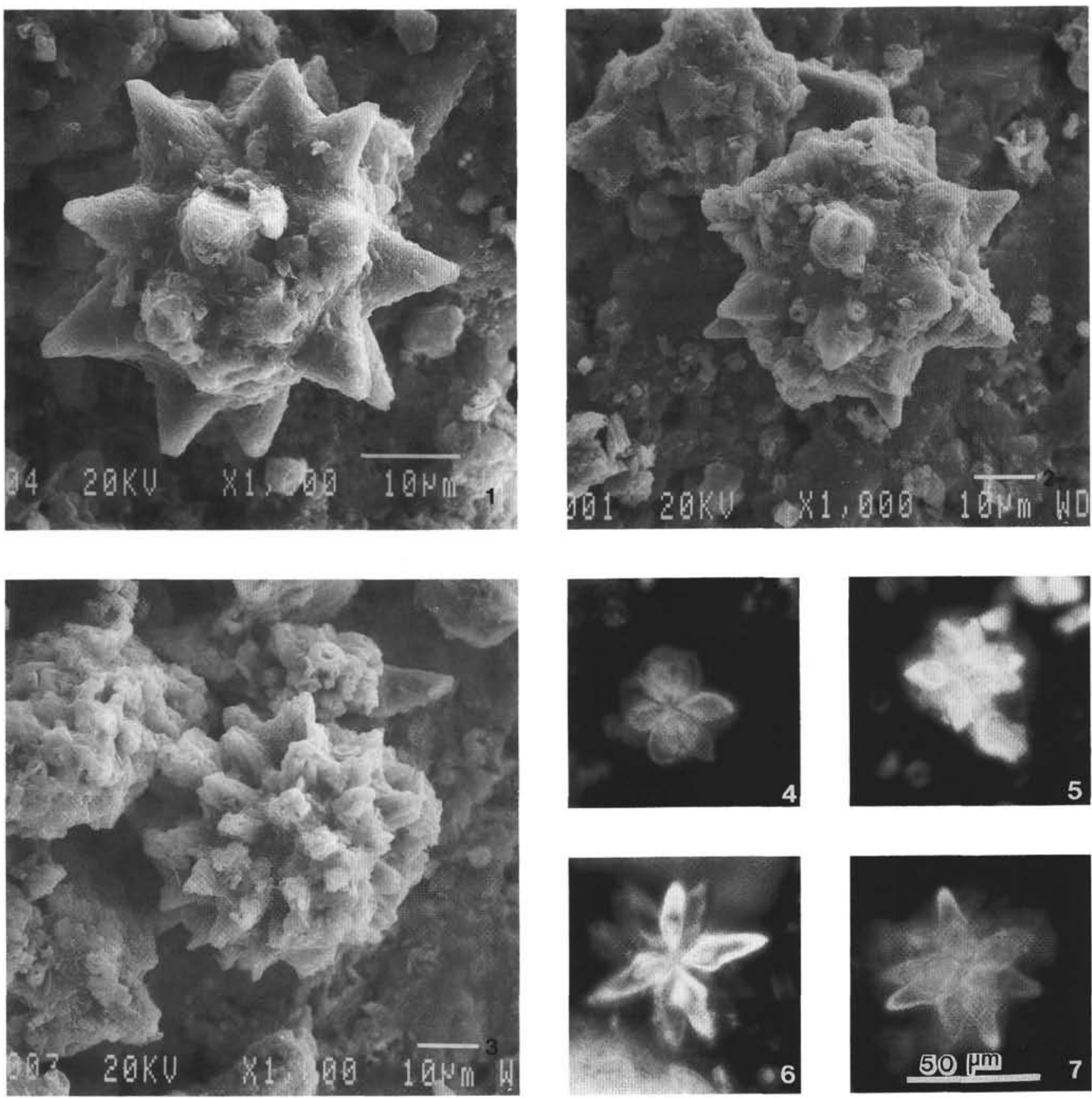

Plate 1. Tunicate spicules, Sample 133-821A-1H-1, $112 \mathrm{~cm}$. 1-3. SEM micrographs, scale bar indicates $10 \mu \mathrm{m}$. 4-7. Light micrographs, same magnification, scale bar indicates $50 \mu \mathrm{m}$. 

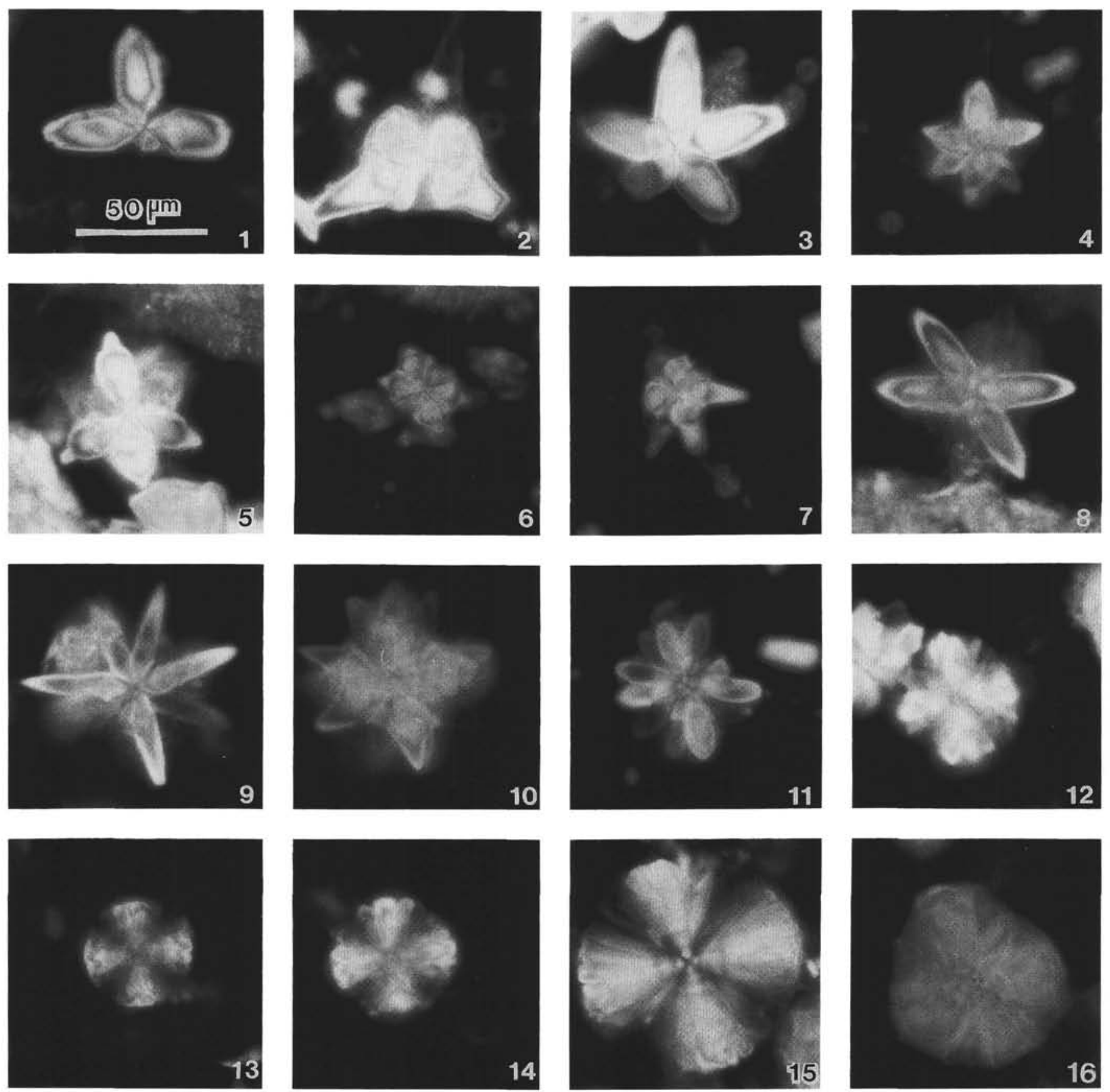

Plate 2. 1-16. Tunicate spicules, Sample $133-821 \mathrm{~A}-1 \mathrm{H}-1,112 \mathrm{~cm}$, light micrographs, same magnification, scale bar indicates $50 \mu \mathrm{m}$. Note variations in the number of rays. 